\title{
INACCESSIBLE BOUNDARY POINTS
}

\author{
A. W. GOODMAN
}

1. Introduction. The Riemann mapping theorem gives immediately the existence of a function $F(z)$, analytic in $E$, the circle $|z|<1$, and mapping that region onto a region having inaccessible boundary points [3, pp. 179-200]. ${ }^{1}$

However, so far as the author is aware, there are no known formal expressions for an $F(z)$ of this type, and it is the purpose of the present work to remedy this defect by giving a class of examples of such functions. As a particular case we shall see that the function

$$
F(z)=\frac{z}{\prod_{n=1}^{\infty}\left(1-z 2 \cos \left(\pi / 2^{(n+1) / 2}\right)+z^{2}\right)^{1 / 2 n}}
$$

maps $E$ onto a region $B_{8}$ of the $w$-plane, for which all of the points $w>8$ are inaccessible boundary points.

2. The slit regions $B_{\imath}$. We shall consider regions formed by deleting from the entire complex plane an infinite number of semi-infinite radial slits, symmetrically placed with respect to the real axis. Because of this symmetry it is sufficient to consider only the slits in the closed upper half-plane. Let $S_{n}$ denote the slit with end point at $w=\rho_{n} e^{i \phi_{n}}$ and suppose the subscripts so chosen that

$$
\pi=\phi_{0}>\phi_{1}>\phi_{2}>\cdots>\phi_{n}>\cdots>\phi_{\infty}=0 .
$$

Definition. A region $B_{s}$ is said to be of type $S$ if it is formed as described above, and if in addition:

(a) There is a constant $m>0$ such that

$$
\begin{aligned}
& m \leqq \rho_{n}<\infty, \quad n=0,1,2, \cdots, \\
& \lim _{n \rightarrow \infty} \phi_{n}=0, \\
& \rho_{\infty}=\liminf _{n \rightarrow \infty} \rho_{n} .
\end{aligned}
$$

It is obvious that if $\rho_{\infty}<\infty$, then all of the points $w>\rho_{\infty}$ are inaccessible boundary points of $B_{s}$. Condition (a) assures the existence of an $F(z)$, such that $F(0)=0, F^{\prime}(0)>0$, and $F(z)$ maps $E$ conformally onto $B_{\mathbf{s}}$. Theorem 1 gives somewhat more information about $F(z)$.

Presented to the Society, September 7, 1951 ; received by the editors July 19, 1951.

1 Numbers in brackets refer to the references at the end of the paper. 
TheOREM 1. For each region $B_{0}$ of type $S$ there are constants $c>0, \theta_{n}$,

$$
\pi>\theta_{1}>\theta_{2}>\cdots>\theta_{n}>\cdots>0, \quad \theta_{n} \rightarrow \theta_{\infty}=0,
$$

such that if

$$
F(z)=\frac{c z}{\prod_{n=1}^{\infty}\left(1-z 2 \cos \theta_{n}+z^{2}\right)^{\gamma_{n}}}
$$

where

$$
\gamma_{n} \pi=\phi_{n-1}-\phi_{n}, \quad n=1,2, \cdots,
$$

then $F(z)$ maps $E$ conformally onto $B_{s}$, with $F(0)=0$ and $F^{\prime}(0)>0$. Conversely each function defined by (2.3) and (2.2) with

$$
\sum_{n=1}^{\infty} \gamma_{n}=1, \quad \gamma_{n}>0 ; n=1,2, \cdots,
$$

maps $E$ conformally onto a region of type $S$ where the directions of the slits are determined by

$$
\phi_{n}=\pi \sum_{j=n+1}^{\infty} \boldsymbol{\gamma}_{j}
$$

Proof. First note that each term of the product

$$
P_{n}(z) \equiv\left(1-z 2 \cos \theta_{n}+z^{2}\right)^{\gamma_{n}}=\left(1-z e^{i \theta_{n}}\right)^{\gamma_{n}}\left(1-z e^{-i \theta_{n}}\right)^{\gamma_{n}}
$$

is to be understood as that branch of the function for which $P_{n}(0)=1$. Let $E_{n}(\delta)$ denote the region obtained by deleting from $E$ the portion common to the circle $\left|z-e^{i \theta_{n}}\right| \leqq \delta$, and the portion common to the circle $\left|z-e^{-i \theta_{n}}\right| \leqq \delta, \delta>0$; and let $E(\delta)$ denote the intersection of the sets $E_{n}(\delta), n=1,2, \cdots$. Then it is clear from (2.7) that in $E_{n}(\delta)$

$$
\begin{aligned}
& \delta^{2 \gamma_{n}} \leqq\left|P_{n}(z)\right| \leqq 4^{\gamma_{n}}, \\
& -\gamma_{n} \pi<\arg P_{n}(z)<\gamma_{n} \pi,
\end{aligned}
$$

and hence for $0<\delta<1$,

$$
\left|\log P_{n}(z)\right| \leqq \gamma_{n}\{\log 4-2 \log \delta+\pi\} .
$$

From (2.5) it follows that in $E(\delta)$ the series

$$
\sum_{n=1}^{\infty} \log P_{n}(z)
$$

converges uniformly and hence by the Weierstrass Theorem repre- 
sents an analytic function in $E(\delta)$. Since $\delta$ can be taken arbitrarily small, the series (2.11) converges in $E$ and hence the product in (2.3) is also convergent in the same region. In fact, the product is uniformly convergent on any closed arc of $|z|=1$ which is free of the points $e^{ \pm i \theta_{n}}, n=1,2, \cdots$.

Suppose now that we have a given fixed region $B_{s}$ of type $S$. By the Riemann mapping theorem, there is a unique function $F_{s}(z)$ mapping $E$ conformally on $B_{s}$, with $F_{s}(0)=0$ and $F_{s}^{\prime}(0)>0$. From the symmetry of $B_{s}, F_{s}(z)$ is real on the real axis and further $r F_{s}(r)>0$ for $0<r^{2}<1$. Denote by $E_{c}$ the region obtained by cutting the open unit circle along the negative real axis, $-1<r \leqq 0$. Then $u_{s}=\arg F_{s}(z)$ is harmonic in $E_{c}$, and further, with a suitable determination, $-\pi$ $\leqq u_{s} \leqq \pi$. A consideration of $1 / F_{s}(z)$ shows that each of the points on the inverted slits $S_{n}^{-1}\left(w=\rho e^{-i \theta_{n}}, 0 \leqq \rho \leqq \rho_{n}^{-1}, 0<\phi \leqq \pi\right)$ is an accessible boundary point and, therefore, the zero of $1 / F_{s}(z)$ corresponding to the vertex of the sector defined by $S_{n}^{-1}$ and $S_{n-1}^{-1}$ is the image of a well-determined point $z=e^{i \theta_{n}}[3, \mathrm{pp} .189-192]$. Even more, the Schwarz reflection principle shows that $z=e^{i \theta_{n}}$ is a simple zero of $\left(F_{s}(z)\right)^{-1 / \gamma_{n}}$. Thus the function $F_{s}(z)$ determines a set of arcs on the boundary of the upper half of the unit circle with end points $e^{i \theta_{n}}$ satisfying (2.2), such that for $\theta_{n+1}<\theta<\theta_{n}$

$$
\arg F_{s}\left(e^{i \theta}\right)=\sum_{j=n+1}^{\infty} \gamma_{j} \pi=\phi_{n}, \quad n=1,2, \cdots .
$$

For $\theta_{1}<\theta<\pi$, arg $F_{s}(z)=\pi$. Finally, if $\theta_{\infty}>0$, then $\arg F_{s}(z)$ can be extended by continuity so that arg $F_{s}\left(e^{i \theta}\right)=0$ for $-\theta_{\infty}<\theta<\theta_{\infty}$. To see this last assertion, observe that any simple curve $\Gamma_{w}$ in $B$, joining $\rho_{n} e^{i \phi_{n}}$ and $\rho_{n} e^{-i \phi_{n}}$ is the image of some simple curve $\Gamma_{z}$ in $E$ joining $e^{i \theta_{n}}$ and $e^{-i \theta_{n}}$. Given $\epsilon>0$, an appropriately chosen $\Gamma_{w}$ with $n$ sufficiently large determines a curve $\Gamma_{z}$, which, together with the arc $\boldsymbol{z}=e^{i \theta},-\theta_{n} \leqq \theta \leqq \theta_{n}$, determines a region in which $\left|\arg F_{s}(z)\right| \leqq \phi_{n}<\epsilon$.

Thus $\arg F_{s}(z)$ is a harmonic function in $E_{c}$, continuous and bounded on the interior, constant on the boundary except for finite jumps of $\pm \gamma_{n} \pi$ at $\pm \theta_{n}$, and a jump of $2 \pi$ at $z=0$.

Next, with the values of $\theta_{n}$ just determined for $F_{s}(z)$, and with the associated values of $\gamma_{n}$, form the function $F(z)$ as in (2.3). If $u$ $\equiv \arg F(z)$ for $z$ in $E_{c}$, then $u$ is also harmonic there and

$$
\begin{aligned}
u & =\arg z-\sum_{j=1}^{\infty} \gamma_{j} \arg \left(1-z 2 \cos \theta_{j}+z^{2}\right) \\
& =\sum_{j=1}^{\infty} \gamma_{j}\left\{\theta-\arg \left(1-z 2 \cos \theta_{j}+z^{2}\right)\right\} .
\end{aligned}
$$


For $z=e^{i \theta}$,

(2.13) $\arg \left(1-z 2 \cos \theta_{j}+z^{2}\right)= \begin{cases}\theta, & \text { if } 0 \leqq \theta<\theta_{j}, \\ \theta-\pi, & \text { if } \theta_{j}<\theta \leqq \pi .\end{cases}$

Thus for the upper half of the boundary of $E_{c}, u$ is a step function, such that for $\theta_{n+1}<\theta<\theta_{n}$

$$
u=\arg F\left(e^{i \theta}\right)=\sum_{j=n+1}^{\infty} \gamma_{j}\{\theta-(\theta-\pi)\}=\phi_{n} .
$$

Similar results hold for the lower half of the boundary of $E_{c}$. Finally when $z=r,-1<r<0, u= \pm \pi$ according as $z$ approaches the boundary from above or below.

Therefore $U=u_{0}-u$ is a bounded harmonic function, continuous in $E_{c}$ and zero on the boundary except for an infinite number of points which have at most two limit points. The conformality of $F(z)$ and $F_{s}(z)$ at the origin permit us to remove the slit, and make the same assertions about $U$ in $E$. It is then easy to see from the Poisson integral formula that $U \equiv 0\left[4\right.$, p. 321]. Thus $F(z)$ and $F_{s}(z)$ differ by at most a multiplicative positive constant. Thus if $c$ is chosen properly in (2.3), $F(z)$ maps $E$ onto the region $B_{\text {. }}$ It is now easy to see that $\theta_{\infty}=0$, for otherwise the arc

$$
z=e^{i \theta}, \quad-\theta_{\infty}<\theta<\theta_{\infty},
$$

would go into a doubly covered slit on the real axis consisting of accessible boundary points. This is indeed a possibility, but we have excluded this possibility by condition (c) of the definition of the region $B_{s}$. If, in (2.3), $\theta_{\infty}>0$ then $F(z)$ will map $E$ onto a region with accessible boundary points on the positive real axis whether or not it has inaccessible boundary points. We have defined $B_{2}$ in such a way as to exclude this occurrence in order to simplify the presentation.

The second part of Theorem 1 will be a trivial consequence of the preceding material as soon as we show that the accessible boundary points of $B_{s}$ form a one-to-one image of the arc $|z|=1, z \neq 1$, under $F(z)$, i.e. as soon as we show that each slit $S_{j}$ is doubly covered. Recalling (2.5) it is easy to see that

$$
\frac{z F^{\prime}(z)}{F(z)}=\sum_{j=1}^{\infty} \frac{\gamma_{j}\left(1-z^{2}\right)}{1-z 2 \cos \theta_{j}+z^{2}} .
$$

Thus $F^{\prime}(-1)=0$, and if $z=1$ is a point of regularity of $F(z)$, i.e. if $\theta_{\infty} \neq 0$, then $F^{\prime}(1)=0$. For $z=e^{i \theta}, \theta_{n+1}<\theta<\theta_{n}$, 


$$
\begin{aligned}
\frac{z F^{\prime}(z)}{F(z)} & =-i \sin \theta\left\{\sum_{j=1}^{n} \frac{\gamma_{j}}{\cos \theta-\cos \theta_{j}}-\sum_{j=n+1}^{\infty} \frac{\gamma_{j}}{\cos \theta_{j}-\cos \theta}\right\} \\
& =-i \sin \theta\{I(\theta)-D(\theta)\}
\end{aligned}
$$

where $I(\theta)$ is an increasing function, tending to $\infty$ as $\theta \rightarrow \theta_{n}$, and $D(\theta)$ is a decreasing function, decreasing from $\infty$ at $\theta=\theta_{n+1}$. Thus in each $\operatorname{arc} \theta_{n+1}<\theta<\theta_{n}, n=1,2, \cdots, F^{\prime}(z)$ has a simple zero which we denote by $z=e^{i \alpha_{n}}$, and $F\left(e^{i \alpha_{n}}\right)=\rho_{n} e^{i \phi_{n}}$, a slit end point.

In case $\theta_{\infty}>0, z=1$ furnishes the simple zero of $F^{\prime}(z)$ for the arc $-\theta_{\infty}<\theta<\theta_{\infty}$, while $z=-1$ is always the simple zero for $\theta_{1}<\theta<2 \pi-\theta_{1}$. The slits $S_{j}$ are doubly covered, and thus Theorem 1 is proved.

We observe that if the region $B_{s}$ is not assumed to be symmetrical, then (2.3) is replaced by

$$
F(z)=\frac{c z}{\prod_{n=1}^{\infty}\left(1-z e^{i \theta_{n}}\right)^{\gamma_{n}}},
$$

where now

$$
\sum_{n=1}^{\infty} \gamma_{n}=2, \quad \gamma_{n}>0
$$

If $B$, has only $m$ slits, then in (2.17) and (2.18) the product and sum have exactly $m$ terms. ${ }^{2}$ In the still more special case that the $e^{i \theta_{n}}$ are taken as the $m$ th roots of unity, and the $\gamma_{n}$ all equal, we obtain the well known

$$
f(z)=\frac{c z}{\left(1-z^{m}\right)^{2 / m}},
$$

which maps the unit circle on the $w$-plane with radial slits whose end points are the vertices of a regular $m$-gon.

Perhaps a more intuitive approach to (2.17) and (2.3) would have been through Alexander's theorem $[1 ; 5, \mathrm{pp} .11-15]$, which states that if

$$
F(z)=z f^{\prime}(z),
$$

then $F(z)$ starlike with respect to the origin implies $f(z)$ convex, and conversely. Then (2.17) and (2.3) would be a simple consequence of

2 After this paper was completed, the author learned from Professor Z. Nehari that formulae (2.17), (2.18), with a finite number of terms, were given earlier by $C$. Darwin [2]. 
the Schwarz-Christoffel transformation

$$
f(z)=c \int_{0}^{s} \frac{d t}{\prod_{n=1}^{\infty}\left(1-t e^{i \theta_{n}}\right)^{\gamma_{n}}} .
$$

It is worth noting that even though $F(z)$ given by (2.17) may map $E$ onto a region with inaccessible boundary points, the associated $f(z)$ given by (2.19) and (2.20) maps $E$ onto a convex region which consequently has no inaccessible boundary points. Thus integration may destroy inaccessible boundary points.

3. Some examples of functions for which $B$, has inaccessible boundary points. In general it may be very difficult to find the zeros of (2.16), but for the problem at hand this is not necessary, since if $\theta_{n+1}<\eta<\theta_{n}$, and if $\alpha_{n}$ is the zero of (2.16) corresponding to this interval, then $\left|F\left(e^{i \alpha_{n}}\right)\right| \leqq\left|F\left(e^{i \eta}\right)\right|$. This together with Theorem 1 gives the following results.

Theorem 2. A necessary and sufficient condition that $F(z)$ given by (2.3) maps $E$ onto a region with inaccessible boundary points is that there is a constant $M$ and a decreasing sequence $\left\{\eta_{n}\right\}$ such that

$$
\lim _{n \rightarrow \infty} \eta_{n}=0 \text {, }
$$

and

$$
\left|F\left(e^{i \eta_{n}}\right)\right| \leqq M, \quad n=1,2, \cdots .
$$

Under these conditions all the points $\omega>M$ will be inaccessible boundary points.

To determine such a sequence we proceed thus. If, in (2.3), $c=1$, then

$$
\log \left|F\left(e^{i \eta}\right)\right|=\log \frac{1}{2}-\sum_{j=1}^{\infty} \gamma_{j} \log \left|\cos \eta-\cos \theta_{j}\right| .
$$

Since $\cos \eta-\cos \theta=-2 \sin (\eta+\theta) / 2 \sin (\eta-\theta) / 2$ and $2|x| / \pi$ $\leqq|\sin x| \leqq|x|$ for $0 \leqq|x| \leqq \pi / 2$, it follows that if $0<\eta, \theta_{j} \leqq \pi / 2$, then

$$
\begin{aligned}
Q(\eta) & \equiv \sum_{j=1}^{\infty} \gamma_{j} \log \left|\cos \eta-\cos \theta_{j}\right| \\
& \geqq \log \frac{2}{\pi^{2}}+\sum_{j=1}^{\infty} \gamma_{i} \log \left|\eta^{2}-\theta_{j}^{2}\right| .
\end{aligned}
$$


Let us suppose that, given $\left\{\gamma_{n}\right\}$ satisfying (2.5), it is possible to determine an increasing sequence of positive constants $\left\{c_{n}\right\}$ such that the series

$$
\begin{aligned}
& \sum_{j=1}^{\infty} e^{-c_{j}}=\sigma, \\
& \sum_{j=1}^{\infty} \gamma_{j} c_{j}=M_{1}
\end{aligned}
$$

both converge. In $F(z)$ take $\theta_{n}>0$ such that

$$
\theta_{n}^{2}=\frac{\pi^{2}}{4 \sigma} \sum_{j=n}^{\infty} e^{-c_{i}}, \quad n=1,2, \cdots,
$$

and note that $\pi / 2=\theta_{1}>\theta_{2}>\cdots>\theta_{\infty}=0$. Next set

$$
\underset{\eta_{n}}{2}=\frac{\hat{\theta}_{n}^{2}+\theta_{n+1}^{2}}{2}=\frac{\pi^{2}}{8 \sigma}\left(e^{-c_{n}}+2 \sum_{j=n+1}^{\infty} e^{-c_{j}}\right)
$$

and observe that $\theta_{1}>\eta_{1}>\theta_{2}>\cdots>\theta_{n}>\eta_{n}>\cdots>0$. Then for all $j, n=1,2 \cdots$,

$$
\left|\eta_{n}^{2}-\theta_{j}^{2}\right| \geqq \frac{\pi^{2}}{8 \sigma} e^{-c_{j}}
$$

Using this in (3.4), and taking into account (3.6), gives

$$
Q\left(\eta_{n}\right)>-M_{1}-\log 4 \sigma .
$$

Consequently from (3.3) and (3.4) we have

$$
\left|F\left(e^{i \eta_{n}}\right)\right|<2 \sigma e^{M_{1}}=M \text {. }
$$

We summarize these results as follows:

LemMA 1. Given $\left\{\gamma_{n}\right\}$ satisfying (2.5), if it is possible to find an increasing sequence of positive constants $\left\{c_{n}\right\}$ such that (3.5) and (3.6) are convergent series, then $F(z)$ defined by (2.3) with $\theta_{n}$ defined by (3.7) maps the unit circle onto a region $B_{8}$ for which all the points $w>2 \sigma e^{M_{1}}$ are inaccessible boundary points.

At first the author conjectured that for every convergent series (2.5) there is a sequence of positive constants $\left\{c_{n}\right\}$ such that (3.5) and (3.6) both converge. Professor James A. Jenkins refuted this conjecture with the following counter example. Set $\gamma_{n}=1 / n(\log n)^{2}$, $n \geqq 2$. If we could find $c_{n}>0$ such that (3.5) and (3.6) both converge, then the sum 


$$
\sum_{2}^{\infty}\left(e^{-c_{n}}+\gamma_{n} c_{n}\right)
$$

would also converge. But this last is impossible, since if $c_{n} \geqq \log n$, then $\gamma_{n} c_{n} \geqq 1 / n \log n$, and if $c_{n} \leqq \log n$, then $e^{-c_{n}} \geqq 1 / n$, so that in any case $e^{-c_{n}}+\gamma_{n} c_{n}>1 / n \log n$.

A rather general case in which Lemma 1 can be applied occurs when $\left\{\gamma_{n}\right\}$ is decreasing and $\sum \gamma_{n} \log n$ converges, for then it suffices to take $c_{n}=-\log \gamma_{n}$ so that (3.5) becomes (2.5). This is possible, for example, when $\gamma_{n}=k n^{-p}, k>0, p>1$.

A still simpler case occurs when $\left\{\gamma_{n}\right\}$ is a geometric sequence, i.e. $\gamma_{n}=A^{n-1}(1-A), 0<A<1$. In this case, taking $c_{n}=-\log A^{n-1}(1-A)$, we have, from (3.5), $\sigma=1$, and from (3.6)

$$
M_{1}=-\frac{A}{1-A} \log A-\log ^{-}(1-A),
$$

so that

$$
M=\frac{2}{(1-A) A^{A /(1-A)}} .
$$

Equation (3.7) gives $\theta_{n}=\pi A^{(n-1) / 2} / 2$. This proves the following theorem.

TheOREM 3. If $0<A<1$, the function

$$
F(z)=\frac{z}{\prod_{n=0}^{\infty}\left(1-z 2 \cos \left(\pi A^{n / 2} / 2\right)+z^{2}\right)^{A^{n}(1-A)}}
$$

maps $E$ onto a region of type $S$ for which all the points $w>M$ are inaccessible boundary points, where $M$ is given by (3.13).

The case $A=1 / 2$ gives the function (1.1) mentioned in the introduction.

4. Further examples. By suitable combinations of $F(z)$ with certain simple functions, other interesting examples can be obtained. Thus if $f_{c}(z)$ maps onto $E$ onto $E_{c}$, then $\log F\left(f_{c}(z)\right)$ maps $E$ onto a strip $|\Im(w)|<\pi$ cut by lines parallel to the real axis in such a way that the points $w>\log M$ are inaccessible boundary points. The function $\left(F^{1 / 2}\left(f_{c}(z)\right)-1\right) /\left(F^{1 / 2}\left(f_{c}(z)\right)+1\right)$ maps $E$ onto the unit circle with circular arc slits so disposed that the points $\left(M^{1 / 2}-1\right) /\left(M^{1 / 2}+1\right)$ $<w \leqq 1$ are inaccessible boundary points. It seems difficult however to give an expression for the function which maps $E$ onto a circle 
with radial slits and having inaccessible boundary points.

The inaccessible boundary points can be rotated by taking $F_{k}(z)$ $=e^{i \delta_{k}} F\left(z e^{-i \delta_{k}}\right)$, and it is then clear that

$$
G_{m}(z)=\left\{\prod_{k=1}^{m} F_{k}(z)\right\}^{1 / m}
$$

maps $E$ onto a region having $m$ radial lines with inaccessible boundary points, providing only that the $\theta_{n}^{(k)}$ for $F_{k}(z)$ satisfy suitable conditions.

Finally if

$$
\begin{aligned}
\frac{\pi}{2}>\theta_{1}>\theta_{2} & >\cdots>\theta_{\infty}>\theta^{*}>0, \\
\gamma^{*}+\sum_{n=1}^{\infty} \gamma_{n}=1, & \gamma^{*}, \gamma_{n}>0,
\end{aligned}
$$

and if the $\theta_{n}$ are suitably chosen, then

$$
F(z)=\frac{z}{\left(1-z 2 \cos \theta^{*}+z^{2}\right)^{\circ} \prod_{n=1}^{\infty}\left(1-z 2 \cos \theta_{n}+z^{2}\right)^{\gamma_{n}}}
$$

maps $E$ onto a region for which points on the two radial lines $w$ $=\rho e^{ \pm i \gamma^{*} \pi}, \rho>M$, are accessible from one side but inaccessible from the other.

\section{REFERENCES}

1. J. W. Alexander, Functions which map the interior of the unit circle upon simple regions, Ann. of Math. vol. 17 (1915) pp. 12-22.

2. C. Darwin, On some transformations involving elliptic functions, Philosophical Magazine (7) vol. 41 (1950) pp. 1-11.

3. L. R. Ford, Automorphic functions, McGraw-Hill, 1929.

4. A. Hurwitz and R. Courant, Funktionentheorie, Springer, 1929.

5. P. Montel, Leģons sur les fonctions univalentes ou multivalentes, GauthierVillars, 1933.

UNIVERSITY OF KENTUCKY 